\title{
BESOV-TYPE CHARACTERISATIONS FOR THE BLOCH SPACE
}

\author{
KAREL StroethofF
}

\begin{abstract}
We will prove local and global Besov-type characterisations for the Bloch space and the little Bloch space. As a special case we obtain that the Bloch space consists of those analytic functions on the unit disc whose restrictions to pseudo-hyperbolic discs (of fixed pseudo-hyperbolic radius) uniformly belong to the Besov space. We also generalise the results to Bloch functions and little Bloch functions on the unit ball in $\mathbb{C}^{m}$. Finally we discuss the related spaces $B M O A$ and $V M O A$.
\end{abstract}

\section{INTRODUCTION}

Let $\mathbf{D}=\{z \in \mathbb{C}:|z|<1\}$ denote the open unit disc in the complex plane. For an analytic function $f$ on $\mathrm{D}$ we set

$$
\|f\|_{\mathcal{B}}=\sup _{z \in \mathbf{D}}\left(1-|z|^{2}\right)\left|f^{\prime}(z)\right|
$$

The Bloch space $\mathcal{B}$ is the set of all analytic functions $f$ on $\mathbf{D}$ for which $\|f\|_{\mathcal{B}}<\infty$. Even though $\|\cdot\|_{B}$ is not a norm, we will refer to $\|f\|_{\mathcal{B}}$ as the Bloch norm of the function $f$. The quantity $|f(0)|+\|f\|_{\mathcal{B}}$ defines a norm on the linear space $\mathcal{B}$ which, equipped with this norm, is a Banach space (see, for example, [1]). For $\lambda \in \mathbf{D}$ let the Möbius function $\varphi_{\lambda}: \mathbf{D} \rightarrow \mathbf{D}$ be defined by

$$
\varphi_{\lambda}(z)=\frac{\lambda-z}{1-\bar{\lambda} z}, z \in \mathbf{D}
$$

For an analytic function $f$ on $\mathrm{D}$ and a point $\lambda \in \mathrm{D}$, we will call $f \circ \varphi_{\lambda}-f(\lambda)$ a Möbius transform of function $f$. An important property of the Bloch space is that it is invariant under Möbius transforms, that is if $f \in \mathcal{B}$ and $\lambda \in \mathrm{D}$, then $f \circ \varphi_{\lambda}-f(\lambda) \in \mathcal{B}$. This is immediate from the definition of the Bloch norm: for analytic $f$ on $\mathbf{D}$ and $\lambda \in \mathbf{D}$

$$
\|f\|_{\mathcal{B}}=\left\|f \circ \varphi_{\lambda}\right\|_{\mathcal{B}} .
$$

In [8] Rubel and Timoney showed that the Bloch space $\mathcal{B}$ is in some sense maximal among all Möbius-invariant Banach spaces of analytic functions on $\mathbf{D}$.

Received 27 July 1988

This paper represents part of the author's Ph.D dissertation written at Michigan State University under the direction of Sheldon Axler.

Copyright Clearance Centre, Inc. Serial-fee code: 0004-9729/89 \$A2.00+0.00. 
For a point $\lambda \in \mathbf{D}$ and $0<r<1$ the pseudo-hyperbolic disc $D(\lambda, r)$ with pseudohyperbolic centre $\lambda$ and pseudo-hyperbolic radius $r$ is defined by $D(\lambda, r)=\varphi_{\lambda}(r \mathrm{D})$. The pseudo-hyperbolic disc $D(\lambda, r)$ is also a euclidean disc: its euclidean centre and euclidean radius are $\left(1-r^{2}\right) \lambda /\left(1-r^{2}|\lambda|^{2}\right)$ and $\left(1-|\lambda|^{2}\right) r /\left(1-r^{2}|\lambda|^{2}\right)$, respectively (see [6]).

Let $A$ denote the normalised Lebesgue area measure on $\mathrm{D}$, and for a Lebesgue measurable set $K \subset \mathrm{D}$, let $|K|$ denote the measure of $K$ with respect to $A$. It follows immediately that:

$$
|D(\lambda, r)|=\frac{\left(1-|\lambda|^{2}\right)^{2}}{\left(1-r^{2}|\lambda|^{2}\right)^{2}} r^{2}
$$

Two quantities $A_{f}$ and $B_{f}$, both depending on an analytic function $f$ on $\mathrm{D}$, are said to be equivalent, written as $A_{f} \approx B_{f}$, if there exists a finite positive constant $C$ not depending on $f$ such that for every analytic function $f$ on $D$ we have:

$$
\frac{1}{C} B_{f} \leqslant A_{f} \leqslant C B_{f}
$$

If the quantities $A_{f}$ and $B_{f}$ are equivalent, then in particular we have $A_{f}<\infty$ if and only if $B_{f}<\infty$.

Our point of departure is the following theorem which is part of a theorem taken from Axler [4], where several other quantities equivalent to the Bloch norm are given.

Theorem I. Let $0<p<\infty$ and let $0<r<1$. Then for an analytic function $f$ on the unit disc $\mathbf{D}$ the following quantities are equivalent:
(A) $\|f\|_{B}$;
(B) $\sup _{\lambda \in \mathrm{D}}\left(\int_{\mathrm{D}}\left|f\left(\varphi_{\lambda}(z)\right)-f(\lambda)\right|^{p} d A(z)\right)^{1 / p}$;
(C) $\sup _{\lambda \in \mathbf{D}}\left(1 /(|D(\lambda, r)|) \int_{D(\lambda, r)}|f(z)-f(\lambda)|^{p} d A(z)\right)^{1 / p}$;
(D) $\sup _{\lambda \in \mathrm{D}}\left(\int_{D(\lambda, r)}\left|f^{\prime}(z)\right|^{2} d A(z)\right)^{1 / 2}$.

Whereas quantities (B) and (C) in Theorem $I$ are expressed for general $p$ in $(0, \infty)$, quantity (D) is given only for the special case that $p=2$. The question arises whether quantity (D) in Theorem I can be replaced by a more general quantity depending upon $p$ and specialising to the above (D) in the case where $p=2$.

Quantities (C) and (D) are local as opposed to quantity (B) which is global; this leads to another question: is there a global version of quantity (D)? 
These questions will be answered in Theorem 1, where we will also give equivalent quantities involving higher derivatives of the function.

Contained in the Bloch space is the little Bloch space $\mathcal{B}_{0}$, which is by definition the set of all analytic functions $f$ on $\mathbf{D}$ for which $\left(1-|z|^{2}\right) f^{\prime}(z) \rightarrow \mathbf{0}$ as $|z| \rightarrow 1-$.

The equivalences of Theorem I carry over to the little Bloch space. Several descriptions of this space are given in the following theorem which is part of a theorem in Axler [4], where one can find several additional characterisations for the little Bloch space.

Theorem II. Let $0<p<\infty$ and let $0<r<1$. Then for an analytic function $f$ on the unit disc $\mathbf{D}$ the following statements are equivalent:

(a) $f \in \mathcal{B}_{0}$;

(b) $\int_{\mathbf{D}}\left|f\left(\varphi_{\lambda}(z)\right)-f(\lambda)\right|^{p} d A(z) \rightarrow 0$ as $|\lambda| \rightarrow 1$;

(c) $1 /(|D(\lambda, r)|) \int_{D(\lambda, r)}|f(z)-f(\lambda)|^{p} d A(z) \rightarrow 0$ as $|\lambda| \rightarrow 1-$;

(d) $\int_{D(\lambda, r)}\left|f^{\prime}(z)\right|^{2} d A(z) \rightarrow 0$ as $|\lambda| \rightarrow 1-$.

The main results of this paper are Theorems 1 and 2 which are stated in the next section. These theorems will be proved in Section 3. In Section 4 we will indicate how the results can be generalised to give characterisations for Bloch functions and little Bloch functions on the unit ball in $C^{m}$. The paper ends with an open question concerning the spaces $B M O A$ and $V M O A$ in Section 5 .

\section{Besov-tyPe Characterisations}

Our main result is the following theorem which relates the Bloch norm of an analytic function with quantities involving integrals of the $n$th derivative of the function.

TheOREM 1. Let $0<p<\infty, 0<r<1$, and $n \in \mathrm{N}$. Then for an analytic function $f$ on $\mathbf{D}$ the following quantities are equivalent:
(A) $\|f\|_{\mathcal{B}}$;
(B) $\sup _{\lambda \in \mathbb{D}}\left(1 /\left(|D(\lambda, r)|^{1-n p / 2}\right) \int_{D(\lambda, r)}\left|f^{(n)}(z)\right|^{p} d A(z)\right)^{1 / p}+\sum_{k=1}^{n-1}\left|f^{(k)}(0)\right|$;
(C) $\sup _{\lambda \in \mathrm{D}}\left(\int_{D(\lambda, r)}\left|f^{(n)}(z)\right|^{p}\left(1-|z|^{2}\right)^{n p-2} d A(z)\right)^{1 / p}+\sum_{k=1}^{n-1}\left|f^{(k)}(0)\right|$;
(D) $\sup _{\lambda \in D}\left(\int_{D}\left|f^{(n)}(z)\right|^{p}\left(1-|z|^{2}\right)^{n p-2}\left(1-\left|\varphi_{\lambda}(z)\right|^{2}\right)^{2} d A(z)\right)^{1 / p}$
$+\sum_{k=1}^{n-1}\left|f^{(k)}(0)\right|$

Remarks. (1) Of special interest are the cases where $n p=2$. For $n=1$ and $p=2$ quantity (B) specialises to quantity (D) of Theorem I, and quantity (C) gives a global 
version of quantity (D) of Theorem I:

$$
\|f\|_{B} \approx \sup _{\lambda \in D}\left(\int_{D}\left|f^{\prime}(z)\right|^{2}\left(1-\left|\varphi_{\lambda}(z)\right|^{2}\right)^{2} d A(z)\right)^{1 / 2} .
$$

(2) For $n=2$ and $p=1$ both quantities (B) and (C) specialise to the local Besov-type equivalence

$$
\|f\|_{B} \approx \sup _{\lambda \in \mathbf{D}} \int_{D(\lambda, r)}\left|f^{\prime \prime}(z)\right| d A(z)+\left|f^{\prime}(0)\right|
$$

The Besov space, the set of analytic functions on the unit disc whose second derivative is integrable over $\mathbf{D}$, is minimal among all Möbius-invariant Banach spaces of analytic functions on $D$ (see [2] or [3]). The above equivalence says that the Bloch space $\mathcal{B}$ is the set of analytic functions on $D$ whose restrictions to pseudo-hyperbolic discs (of a fixed pseudo-hyperbolic radius) are uniformly in the Besov space.

For $n=2$ and $p=1$ quantity (D) specialises to the global Besov-type equivalence

$$
\|f\|_{\mathcal{B}} \approx \sup _{\lambda \in \mathbf{D}} \int_{\mathbf{D}}\left|f^{\prime \prime}(z)\right|\left(1-\left|\varphi_{\lambda}(z)\right|^{2}\right)^{2} d A(z)+\left|f^{\prime}(0)\right|
$$

(3) In the case that $n=1$ quantities (C) and (D) are of interest because the quantity

$$
\int_{\mathbf{D}}\left|f^{\prime}(z)\right|^{p}\left(1-|z|^{2}\right)^{p-2} d A(z)
$$

is invariant under Möbius transformations of $f$.

The equivalences of Theorem 1 carry over to the little Bloch space, as is shown in the following theorem.

Theorem 2. Let $0<p<\infty, 0<r<1$, and $n \in \mathbb{N}$. Then for an analytic function $f$ on $D$ the following statements are equivalent:
(a) $f \in \mathcal{B}_{0}$;
(b) $1 /\left(|D(\lambda, r)|^{1-n p / 2}\right) \int_{D(\lambda, r)}\left|f^{(n)}(z)\right|^{p} d A(z) \rightarrow 0$ as $|\lambda| \rightarrow 1-$;
(c) $\int_{D(\lambda, r)}\left|f^{(n)}(z)\right|^{p}\left(1-|z|^{2}\right)^{n p-2} d A(z) \rightarrow 0$ as $|\lambda| \rightarrow 1-$;
(d) $\int_{\mathbf{0}}\left|f^{(n)}(z)\right|^{p}\left(1-|z|^{2}\right)^{n p-2}\left(1-\left|\varphi_{\lambda}(z)\right|^{2}\right)^{2} d A(z) \rightarrow 0$ as $|\lambda| \rightarrow 1-$.

\section{ProOfS}

The proof of Theorem 1 will make use of the following lemma, which relates the Bloch norm of an analytic function with quantities involving higher derivatives. 
LEMma 3. Let $n \in N$. Then for an analytic function $f$ on $\mathbf{D}$ the following quantities are equivalent:

(A) $\|f\|_{B}$;

(B) $\sup _{z \in \mathbf{D}}\left(1-|z|^{2}\right)^{n}\left|f^{(n)}(z)\right|+\sum_{k=1}^{n-1}\left|f^{(k)}(0)\right|$.

Proof: For $n=1$ the equivalence of the two quantities is precisely the definition of the Bloch norm. By induction it suffices to show that for a fixed $n \in N$, for every analytic function $f$ on $\mathrm{D}$ the quantities

$$
\left(\mathbf{B}_{n}\right) \sup _{z \in \mathbf{0}}\left(1-|z|^{2}\right)^{n}\left|f^{(n)}(z)\right|+\sum_{k=1}^{n-1}\left|f^{(k)}(0)\right|
$$

and

$$
\left(B_{n+1}\right) \sup _{z \in \mathbf{D}}\left(1-|z|^{2}\right)^{n+1}\left|f^{(n+1)}(z)\right|+\sum_{k=1}^{n}\left|f^{(k)}(0)\right|
$$

are equivalent.

For $w \in \mathrm{D}$ we have:

$$
\begin{aligned}
\left|f^{(n)}(w)-f^{(n)}(0)\right| & \leqslant \int_{0}^{1}|w|\left|f^{(n+1)}(t w)\right| d t \\
& \leqslant \int_{0}^{1} \frac{|w|}{(1-t|w|)^{n+1}} d t \cdot \sup _{z \in \mathbb{D}}(1-|z|)^{n+1}\left|f^{(n+1)}(z)\right| \\
& \leqslant \frac{1}{n(1-|w|)^{n}} \sup _{n \in \mathbb{D}}(1-|z|)^{n+1}\left|f^{(n+1)}(z)\right| .
\end{aligned}
$$

Multiply by $\left(1-|w|^{2}\right)^{n}$ and take the supremum over all $w \in \mathbf{D}$, to get

$$
\sup _{w \in \mathbf{D}}\left(1-|w|^{2}\right)^{n}\left|f^{(n)}(w)\right| \leqslant \frac{2^{n}}{n} \sup _{z \in \mathbf{D}}\left(1-|z|^{2}\right)^{n+1}\left|f^{(n+1)}(z)\right|+\left|f^{(n)}(0)\right| .
$$

Hence quantity $\left(\mathrm{B}_{n}\right)$ is less than or equal to $2^{n} / n$ times $\left(\mathrm{B}_{n+1}\right)$.

For the converse, fix $z \in D$ and put $r=(1-|z|) / 2$. It is an easy consequence of the Cauchy Integral Formula for the derivative of an analytic function that

$$
\left|f^{(n+1)}(z)\right| \leqslant \frac{1}{r} \sup \left\{\left|f^{(n)}(w)\right|:|w-z|=r\right\} .
$$

If $|w-z|=r$, then $|w| \leqslant|z|+r=(1+|z|) / 2$, so that by the maximum modulus principle $\sup \left\{\left|f^{(n)}(w)\right|:|w-z|=r\right\} \leqslant \sup \left\{\left|f^{(n)}(w)\right|:|w|=(1+|z|) / 2\right\}$. Multiply both sides of inequality (3) by $(1-|z|)^{n+1}=2^{n+1} r^{n+1}$ to get $(1-|z|)^{n+1}\left|f^{(n+1)}(z)\right| \leqslant$ $2^{n+1} \sup \left\{r^{n}\left|f^{(n)}(w)\right|:|w|=(1+|z|) / 2\right\}$. For $|w|=(1+|z|) / 2$ we have $r=1-|w| \leqslant$ $1-|w|^{2}$, so after we multiply by $(1+|z|)^{n+1}$ (which is less than $2^{n+1}$ ), we get

$$
\left(1-|z|^{2}\right)^{n+1}\left|f^{(n+1)}(z)\right| \leqslant 2^{2 n+2} \sup \left\{\left(1-|w|^{2}\right)^{n}\left|f^{(n)}(w)\right|:|w|=(1+|z|) / 2\right\} \text {. }
$$


Hence quantity $\left(B_{n+1}\right)$ is less than or equal to $2^{2 n+2}$ times quantity $\left(B_{n}\right)$. This completes the induction and the lemma is proved.

The equivalences of Lemma 3 carry over to the little Bloch space, as is shown in the following lemma.

LEMma 4. Let $n \in N$. Then for an analytic function $f$ on $\mathbf{D}$ the following statements are equivalent:

(a) $f \in \mathcal{B}_{0}$;

(b) $\left(1-|z|^{2}\right)^{n} f^{(n)}(z) \rightarrow 0$ as $|z| \rightarrow 1-$.

ProOF: For $n=1$ the equivalence of the two statements is precisely the definition of the little Bloch space. By induction it suffices to show that for a fixed $n \in \mathbf{N}$, for every analytic function $f$ on $D$ the statements $\left(b_{n}\right)\left(1-|z|^{2}\right)^{n} f^{(n)}(z) \rightarrow 0$ as $|z| \rightarrow 1-$ and $\left(b_{n+1}\right)\left(1-|z|^{2}\right)^{n+1} f^{(n+1)}(z) \rightarrow 0$ as $|z| \rightarrow 1-$ are equivalent.

Let $n \in \mathrm{N}$ be fixed. That statement $\left(b_{n}\right)$ implies statement $\left(b_{n+1}\right)$ follows easily from (4). For the converse suppose that $f$ is an analytic function on $D$ satisfying condition $\left(b_{n+1}\right)$. Let $0<r<1$. Then as in the proof of Lemma 3 , for $w \in \mathbf{D}$ :

$$
\left|f^{(n)}(w)-f^{(n)}(r w)\right| \leqslant \frac{1}{n(1-|w|)^{n}} \sup \left\{(1-|z|)^{n+1}\left|f^{(n+1)}(z)\right|: r|w| \leqslant|z|<1\right\} .
$$

For $\varepsilon>0$, choose $r \in(0,1)$ such that $\left(1-|z|^{2}\right)^{n+1}\left|f^{(n+1)}(z)\right|<\varepsilon$ whenever $r^{2}<|z|<1$. It follows from the above inequality that $(1-|w|)^{n}\left|f^{(n)}(w)\right| \leqslant$ $\varepsilon / n+(1-|w|)^{n}\left|f^{(n)}(r w)\right|$ whenever $r<|w|<1$. Hence $\left(1-|w|^{2}\right)^{n} f^{(n)}(w) \rightarrow 0$ as $|w| \rightarrow 1-$, that is, $f$ satisfies $\left(b_{n}\right)$. This completes the induction, and the lemma is proved.

Before proving Theorems 1 and 2 we recall a few facts about the Möbius functions $\varphi_{\lambda}$. First, the function $\varphi_{\lambda}$ is easily seen to be it own inverse under composition:

$$
\left(\varphi_{\lambda} \circ \varphi_{\lambda}\right)(z)=z \text { for all } z \in \mathbf{D} \text {. }
$$

The following identity can be obtained by straight forward computation:

$$
1-\left|\varphi_{\lambda}(z)\right|^{2}=\frac{\left(1-|\lambda|^{2}\right)\left(1-|z|^{2}\right)}{|1-\bar{\lambda} z|^{2}},(\lambda, z \in \mathbf{D}) .
$$

A slightly different form in which we will apply the above identity is:

$$
\frac{1-\left|\varphi_{\lambda}(z)\right|^{2}}{1-|z|^{2}}=\left|\varphi_{\lambda}^{\prime}(z)\right|,(\lambda, z \in \mathbf{D}) \text {. }
$$


For $\lambda \in \mathbf{D}$, the substitution $z=\varphi_{\lambda}(w)$ results in the Jacobian change in measure given by $d A(w)=\left|\varphi_{\lambda}^{\prime}(z)\right|^{2} d A(z)$. For a Lebesgue integrable or a non-negative Lebesgue measurable function $h$ on $\mathbf{D}$ we thus have the following change-of-variable formula:

$$
\int_{D(0, r)} h\left(\varphi_{\lambda}(w)\right) d A(w)=\int_{D(\lambda, r)} h(z)\left(\frac{1-\left|\varphi_{\lambda}(z)\right|^{2}}{1-|z|^{2}}\right)^{2} d A(z)
$$

Proof of Theorem 1: Take $0<p<\infty, 0<r<1$, and $n \in N$. By subharmonicity we have that for an analytic function $g$ on $D$ :

$$
|g(0)|^{p} \leqslant \frac{1}{r^{2}} \int_{D(0, r)}|g(w)|^{p} d A(w) .
$$

Let $f$ be an analytic function on $\mathbf{D}$, and let $\lambda \in \mathbf{D}$. Applying the above inequality to the function $g=f^{(n)} \circ \varphi_{\lambda}$, and using charge-of-variable formula (6), we get

$$
\begin{aligned}
\left|f^{(n)}(\lambda)\right|^{p} & \leqslant \frac{1}{r^{2}} \int_{D(0, r)}\left|f^{(n)}\left(\varphi_{\lambda}(w)\right)\right|^{p} d A(w) \\
& =\frac{1}{r^{2}} \int_{D(\lambda, r)}\left|f^{(n)}(z)\right|^{p}\left(\frac{1-\left|\varphi_{\lambda}(z)\right|^{2}}{1-|z|^{2}}\right)^{2} d A(z) \\
& \leqslant \frac{16}{r^{2}\left(1-|\lambda|^{2}\right)^{2}} \int_{D(\lambda, r)}\left|f^{(n)}(z)\right|^{p} d A(z),
\end{aligned}
$$

where we used that $\left(1-\left|\varphi_{\lambda}(z)\right|^{2}\right) /\left(1-|z|^{2}\right) \leqslant 4 /\left(1-|\lambda|^{2}\right)$ for $z \in D$. Using (1) it follows that

$$
\left(1-|\lambda|^{2}\right)^{n}\left|f^{(n)}(\lambda)\right| \leqslant \frac{4^{2 / p}}{r^{n}\left(1-r^{2}\right)^{2 / p}}\left(\frac{1}{|D(\lambda, r)|^{1-n p / 2}} \int_{D(\lambda, r)}\left|f^{(n)}(z)\right|^{p} d A(z)\right)^{1 / p} \text {. }
$$

By Lemma 3 it follows that quantity (A) is less than or equal to $C$ times quantity (B).

That quantities (B) and (C) are equivalent is an immediate consequence of the fact that $\left(1-|z|^{2}\right)^{2} \approx|D(\lambda, r)|$, whenever $z \in D(\lambda, r)$.

For $z \in D(\lambda, r)$ we have $\left(1-\left|\varphi_{\lambda}(z)\right|^{2}\right)^{2}>\left(1-r^{2}\right)^{2}$, thus

$$
\begin{aligned}
\int_{\mathbf{D}}\left|f^{(n)}(z)\right|^{p} & \left(1-|z|^{2}\right)^{n p-2}\left(1-\left|\varphi_{\lambda}(z)\right|^{2}\right)^{2} d A(z) \\
& \geqslant\left(1-r^{2}\right)^{2} \int_{D(\lambda, r)}\left|f^{(n)}(z)\right|^{p}\left(1-|z|^{2}\right)^{n p-2} d A(z)
\end{aligned}
$$


and if follows that quantity (D) is greater than or equal to a constant times quantity (C). To complete the proof we will show that quantity (D) is less than or equal a constant times quantity (A). Again we make use of Lemma 3.

$$
\begin{aligned}
\int_{\mathbf{D}}\left|f^{(n)}(z)\right|^{p} & \left(1-|z|^{2}\right)^{n p-2}\left(1-\left|\varphi_{\lambda}(z)\right|^{2}\right)^{2} d A(z) \\
& \leqslant\left(\sup _{z \in \mathbf{D}}\left(1-|z|^{2}\right)^{n}\left|f^{(n)}(z)\right|\right)^{p} \int_{\mathbf{D}}\left(\frac{1-\left|\varphi_{\lambda}(z)\right|^{2}}{1-|z|^{2}}\right)^{2} d A(z) \\
& =\left(\sup _{z \in \mathbf{D}}\left(1-|z|^{2}\right)^{n}\left|f^{(n)}(z)\right|\right)^{p} .
\end{aligned}
$$

Thus quantity (D) is less than or equal to quantity (B) of Lemma 3 , and the proof is complete.

Proof of Theorem 2: Take $0<p<\infty, 0<r<1$, and $n \in N$. Let $f$ be an analytic function on $D$. That (d) implies (c) is a consequence of inequality (8). The equivalence of statements (b) and (c) follows immediately from the fact that for $z \in$ $D(\lambda, r)$ we have $\left(1-|z|^{2}\right)^{2} \approx|D(\lambda, r)|$. Inequality (7) together with Lemma 4 give that (b) implies (a). To complete the proof we will show that (a) implies (d). Suppose that (a) holds, that is $f \in \mathcal{B}_{0}$. By Lemma 4 we have that $\left(1-|z|^{2}\right)^{n}\left|f^{(n)}(z)\right| \rightarrow 0$ as $|z| \rightarrow 1-$. Then it is easy to see that

$$
\int_{\mathbf{D} \backslash \rho \mathbf{D}}\left|f^{(n)}(z)\right|^{p}\left(1-|z|^{2}\right)^{n p-2}\left(1-\left|\varphi_{\lambda}(z)\right|^{2}\right)^{2} d A(z) \rightarrow 0 \text { as } \rho \rightarrow 1-\text {. }
$$

uniformly in $\lambda$. By Lemma 3 there is a constant $C$ such that $\left(1-|z|^{2}\right)^{n}\left|f^{(n)}(z)\right| \leqslant C$ for every $z \in \mathbf{D}$. It follows from

$$
\begin{aligned}
\int_{\rho \mathbf{D}}\left|f^{(n)}(z)\right|^{p} & \left(1-|z|^{2}\right)^{n p-2}\left(1-\left|\varphi_{\lambda}(z)\right|^{2}\right)^{2} d A(z) \\
& \leqslant C^{p} \int_{D(0, \rho)}\left(\frac{1-\left|\varphi_{\lambda}(z)\right|^{2}}{1-|z|^{2}}\right)^{2} d A(z)=C^{p}|D(\lambda, \rho)|
\end{aligned}
$$

that for every $\rho \in(0,1)$ we have

$$
\int_{\rho \mathbf{D}}\left|f^{(n)}(z)\right|^{p}\left(1-|z|^{2}\right)^{n p-2}\left(1-\left|\varphi_{\lambda}(z)\right|^{2}\right)^{2} d A(z) \rightarrow 0 \text { as }|\lambda| \rightarrow 1-
$$

Combining (9) and (10) yields that (d) holds. 


\section{Generalisations to the unit ball in $\mathbf{C}^{m}$}

Let $m \in \mathrm{N}$ be fixed. For $z, w \in \mathrm{C}^{m}$ let

$$
\langle z, w\rangle=\sum_{k=1}^{m} z_{k} \overline{w_{k}}, \text { and let }\|z\|=\langle z, z\rangle^{1 / 2} \text {. }
$$

Let $\mathbf{B}_{\boldsymbol{m}}=\left\{z \in \mathbb{C}^{m}:\|z\|<1\right\}$ denote the open unit ball. For a holomorphic function $f: B_{m} \rightarrow C$ we set

$$
\|f\|_{\mathcal{B}\left(\mathbf{B}_{m}\right)}=\sup _{z \in \mathbf{B}_{m}}\left(1-\|z\|^{2}\right)\|\nabla f(z)\|
$$

where $\nabla f=\left(\partial f / \partial z_{1}, \ldots, \partial f / \partial z_{m}\right)$ is the gradient of $f$. The Bloch space $\mathcal{B}\left(\mathrm{B}_{m}\right)$ is the set of all holomorphic functions $f: \mathbf{B}_{m} \rightarrow C$ for which $\|f\|_{B\left(B_{m}\right)}<\infty$. The quantity $|f(0)|+\|f\|_{\mathcal{B}\left(\mathbf{B}_{m}\right)}$ defines a norm on the linear space $\mathcal{B}\left(\mathbf{B}_{m}\right)$ which, equipped with this norm, is a Banach space (see [10]).

For a multi-index $\alpha=\left(\alpha_{1}, \ldots, \alpha_{m}\right)$ (each $\alpha_{k}$ a nonnegative integer) we write $|\alpha|=\alpha_{1}+\ldots+\alpha_{m}$, and for a holomorphic function $f: \mathbf{B}_{m} \rightarrow \mathbb{C}$ we write

$$
\frac{\partial^{|\alpha|} f}{\partial z^{\alpha}}=\frac{\partial^{|\alpha|} f}{\partial z_{1}^{\alpha_{1}} \ldots \partial z_{m}^{\alpha_{m}}}
$$

A generalisation of Lemma 3 is the following result which was recently obtained by Zhu [11]. His proof relies heavily on the boundedness of the Bergman projection. We will indicate how the proof of Lemma 3 can be adjusted to give the result.

Lemma 5. Let $n \in \mathrm{N}$. Then for a holomorphic function $f: \mathbf{B}_{m} \rightarrow \mathrm{C}$ the following quantities are equivalent:

(A) $\|f\|_{\mathbf{B}\left(\mathbf{B}_{m}\right)}$;

(B) $\max _{|\alpha|=n} \sup _{z \in \mathbf{B}_{m}}\left(1-\|z\|^{2}\right)^{n}\left|\frac{\partial^{n} f}{\partial z^{\alpha}}(z)\right|+\sum_{|\beta|<n}\left|\frac{\theta^{|\rho|} f}{\partial z^{\beta}}(0)\right|$.

Proof: For $n=1$ the equivalence of the two quantities follows from the definition of the Bloch norm and the inequalities

$$
\max _{1 \leqslant k \leqslant m}\left|\frac{\partial f}{\partial z_{k}}(z)\right| \leqslant\|\nabla f(z)\| \leqslant \sqrt{m} \max _{1 \leqslant k \leqslant m}\left|\frac{\partial f}{\partial z_{k}}(z)\right|, z \in \mathbf{B}_{m}
$$

By induction on $n$ it suffices to show that for a given multi-index $\alpha$ with $|\alpha|=n$ we have the equivalence

$$
\sup _{z \in \mathbf{B}_{m}}\left(1-\|z\|^{2}\right)^{n}\left|\frac{\partial^{n} f}{\partial z^{\alpha}}(z)\right| \approx \max _{1 \leqslant k \leqslant m} \sup _{z \in \mathbf{B}_{m}}\left(1-\|z\|^{2}\right)^{n+1}\left|\frac{\partial^{n+1} f}{\partial z_{k} \partial z^{\alpha}}(z)\right|+\left|\frac{\partial^{n} f}{\partial z^{\alpha}}(0)\right| .
$$


In the proof of Lemma 3 replace (2) by

$$
\left|\frac{\partial^{n} f}{\partial z^{\alpha}}(w)-\frac{\partial^{n} f}{\partial z^{\alpha}}(0)\right| \leqslant \sum_{k=1}^{m} \int_{0}^{1}|w|\left|\frac{\partial^{n+1} f}{\partial z_{k} \partial z^{\alpha}}(t w)\right| d t
$$

which holds for all space $w \in \mathbf{B}_{\boldsymbol{m}}$, to arrive at the inequality

$$
\begin{aligned}
\sup _{w \in \mathbf{B}_{m}}\left(1-\|w\|^{2}\right)^{n}\left|\frac{\partial^{n} f}{\partial z^{\alpha}}(w)\right| \leqslant & \frac{m 2^{n}}{n} \max _{1 \leqslant k \leqslant m} \sup _{z \in \mathbf{B}_{m}}\left(1-\|z\|^{2}\right)^{n+1}\left|\frac{\partial^{n+1} f}{\partial z_{k} \partial z^{\alpha}}(z)\right| \\
& +i\left|\frac{\partial^{n} f}{\partial z^{\alpha}}(0)\right| .
\end{aligned}
$$

For the converse, fix $z \in \mathbf{B}_{m}$, and let $r=(1-\|z\|) / 2$. By inequality (3)

$$
\left|\frac{\partial^{n+1} f}{\partial z_{k} \partial z^{\alpha}}(z)\right| \leqslant \frac{1}{r} \sup \left\{\left|\frac{\partial^{n} f}{\partial z^{\alpha}}(w)\right|: w_{j}=z_{j}(j \neq k),\left|w_{k}-z_{k}\right|=r\right\} .
$$

If $w_{j}=z_{j}(j \neq k)$ and $\left|w_{k}-z_{k}\right|=r$, then it is easily shown that $\|w\| \leqslant\|z\|+r$, so that by the same arguments as in the proof of Lemma 3 we get

$$
\left(1-\|z\|^{2}\right)^{n+1}\left|\frac{\partial^{n+1} f}{\partial z_{k} \partial z^{\alpha}}(z)\right| \leqslant 2^{2 n+2} \sup \left\{\left(1-\|w\|^{2}\right)^{n}\left|\frac{\partial^{n} f}{\partial z^{\alpha}}(w)\right|:\|w\|=(1+\|z\|) / 2\right\},
$$

and the proof of the lemma is complete.

Contained in the Bloch space is the little Bloch space $\mathcal{B}_{0}\left(\mathbf{B}_{m}\right)$, the set of all holomorphic functions $f: B_{m} \rightarrow C$ for which $\left(1-\|z\|^{2}\right)\|\nabla f(z)\| \rightarrow 0$ as $\|z\| \rightarrow 1-$. The equivalences of Lemma 5 carry over to the little Bloch space, as is shown in the following lemma. Since the proof is similar to that of Lemma 4 we omit it.

Lemma 6. Let $n \in \mathbf{N}$. Then for a holomorphic function $f: \mathbf{B}_{m} \rightarrow \mathbb{C}$ the following statements are equivalent:

(a) $f \in \mathcal{B}_{0}\left(\mathbf{B}_{m}\right)$;

(b) $\left(1-\|z\|^{2}\right)^{n} \frac{\theta^{n} f}{\partial z^{\alpha}}(z) \rightarrow 0$ as $\|z\| \rightarrow 1-(|\alpha|=n)$.

For $\lambda \in \mathbf{B}_{m}$ let the Möbius transformation $\varphi_{\lambda}: \mathbf{B}_{n_{2}} \rightarrow \mathbf{B}_{n_{2}}$ be defined by

$$
\varphi_{\lambda}(z)=\frac{\lambda-P_{\lambda} z-\left(1-\|\lambda\|^{2}\right)^{1 / 2} Q_{\lambda} z}{1-\langle z, \lambda\rangle}
$$

where $P_{\lambda} z=\langle z, \lambda\rangle \lambda /\|\lambda\|^{2}$, and $Q_{\lambda} z=z-P_{\lambda} z\left(z \in \mathbf{B}_{m}\right)$. As in the one-dimensional case $\mathcal{B}\left(\mathbf{B}_{\boldsymbol{m}}\right)$ is invariant under Möbius transforms, that is, if $f \in \mathcal{B}\left(\mathbf{B}_{\boldsymbol{m}}\right)$ and $\lambda \in \mathbf{B}_{\boldsymbol{m}}$, 
then $f \circ \varphi_{\lambda}-f(\lambda) \in \mathcal{B}\left(\mathbf{B}_{m}\right)$. This is surprising since the Bloch norm as defined above does not appear to be invariant under Möbius transforms (see [10]).

For a point $\lambda \in \mathbf{B}_{m}$ and $0<r<1$ the pseudo-hyperbolic ball $B(\lambda, r)$ with pseudo-hyperbolic centre $\lambda$ and pseudo-hyperbolic radius $r$ is defined by $B(\lambda, r)=$ $\varphi_{\lambda}\left(r \mathbf{B}_{\mathrm{m}}\right)$. The pseudo-hyperbolic ball $B(\lambda, r)$ is an ellipsoid (see Section 2.2.7 in [0]).

Let $V$ denote the normalised Lebesgue measure on $\mathbf{B}_{m}$ and for a Lebesgue measurable set $K \subset \mathbf{B}_{m}$, let $|K|$ denote the measure of $K$ with respect to $V$. In Section 2.2.7 of $[9]$ it is shown that

$$
|B(\lambda, r)|=\left(\frac{1-\|\lambda\|^{2}}{1-r^{2}\|\lambda\|^{2}}\right)^{m+1} r^{2 m}
$$

As in the one-dimensional case, the map $\varphi_{\lambda}$ is easily seen to be it own inverse under composition: $\left(\varphi_{\lambda} \circ \varphi_{\lambda}\right)(z)=z$ for all $z \in \mathbf{B}_{m}$. Even though the Möbius transformations are far more complicated than in the one-dimensional case, the following identity holds:

$$
1-\left\|\varphi_{\lambda}(z)\right\|^{2}=\frac{\left(1-\|\lambda\|^{2}\right)\left(1-\|z\|^{2}\right)}{|1-\langle z, \lambda\rangle|^{2}},\left(z, \lambda \in \mathbf{B}_{n}\right) .
$$

The real Jacobian of $\varphi_{\lambda}$ at $z \in \mathrm{B}_{m}$ is

$$
\begin{aligned}
\left(J_{\mathbf{R}} \varphi_{\lambda}\right)(z) & =\left(\frac{1-\|\lambda\|^{2}}{|1-\langle z, \lambda\rangle|^{2}}\right)^{m+1} \\
& =\left(\frac{1-\left\|\varphi_{\lambda}(z)\right\|^{2}}{1-\|z\|^{2}}\right)^{m+1}
\end{aligned}
$$

Thus, for a Lebesgue integrable or non-negative Lebesgue measurable function $h$ on $\mathbf{B}_{m}$ we have the change-of-variable formula:

$$
\int_{B(0, r)} h\left(\varphi_{\lambda}(w)\right) d V(w)=\int_{B(\lambda, r)} h(z)\left(\frac{1-\left\|\varphi_{\lambda}(z)\right\|^{2}}{1-\|z\|^{2}}\right)^{n+1} d V(z) .
$$

Using change-of-variable formula (11) instead of (6) and Lemma 5 instead of Lemma 3 in the proof of Theorem 1 it can be shown that this theorem generalises to the following result:

Theorem 7. Let $0<p<\infty, 0<r<1$, and $n \in \mathbf{N}$. Then for a holomorphic function $f: \mathbf{B}_{m} \rightarrow \mathbf{C}$ the following quantities are equivalent:

$$
\text { (A) }\|f\|_{\mathcal{B}\left(\mathbf{B}_{m}\right)} \text {; }
$$


(B) $\max _{|\alpha|=n} \sup _{\lambda \in \mathbf{B}_{m}}\left(\frac{1}{|B(\lambda, r)|^{1-n p /(r n+1)}} \int_{B(\lambda, r)}\left|\frac{\theta^{n} f}{\partial z^{\alpha}}(z)\right|^{p} d V(z)\right)^{1 / p}$

$+\sum_{|\beta|<n}\left|\frac{\partial^{|\beta|} f}{\partial z^{\beta}}(0)\right|$

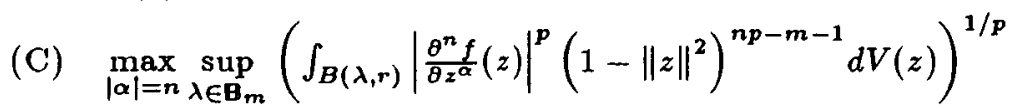

$+\sum_{|\beta|<n}\left|\frac{\partial^{|\beta|} f}{\partial x^{\beta}}(0)\right|:$

(D) $\max _{|\alpha|=n} \sup _{\lambda \in \mathbf{B}_{m}}\left(\int_{\mathbf{B}_{m}}\left|\frac{\theta^{n} f}{\partial z^{\alpha}}(z)\right|^{p}\left(1-\|z\|^{2}\right)^{n p-m-1}\left(1-\left\|\varphi_{\lambda}(z)\right\|^{2}\right)^{m+1} d V(z)\right)^{1 / p}$

$+\sum_{|\beta|<n}\left|\frac{\theta^{|\beta|} f}{\partial z^{\beta}}(0)\right|$.

Remark. Of special interest are the cases where $n p=m+1$. Then both quantities (B) and (C) give the following equivalence:

$$
\begin{aligned}
\|f\|_{\mathcal{B}\left(\mathbf{B}_{m}\right)} \approx \max _{|\alpha|=n} \sup _{\lambda \in \mathbf{B}_{m}}\left(\int_{B(\lambda, r)}\left|\frac{\partial^{n} f}{\partial z^{\alpha}}(z)\right|^{p} d V(z)\right)^{1 / p} \\
+\sum_{|\beta|<n}\left|\frac{\partial^{|\beta|} f}{\partial z^{\beta}}(0)\right| .
\end{aligned}
$$

The theorem and the above equivalence say then that the Bloch space $\mathcal{B}\left(\boldsymbol{B}_{m}\right)$ is the set of holomorplic functions $f: \mathbf{B}_{m} \rightarrow \mathrm{C}$ whose restrictions to pseudo-hyperbolic balls (of a fixed pseudo-hyperbolic radius) are uniformly in the Sobolev p-space of order $n$. (the set of those functions whose partial derivatives of order less than or equal $n$ are $p$-integrable).

It is clear that the equivalences in the above theorem carry over to the little Bloch space to give the following result:

Theorem 8. Let $0<p<\infty, 0<r<1$, and $n \in \mathbf{N}$. Then for a holomorphic function $f: \mathbf{B}_{m} \rightarrow \mathrm{C}$ the following statements are equivalent:

(a) $f \in \mathcal{B}_{0}\left(\mathbf{B}_{m}\right)$;

(b) $\frac{1}{|B(\lambda, r)|^{1-n p /(m+1)}} \int_{B(\lambda, r)}\left|\frac{\partial^{n} f}{\partial z^{\alpha}}(z)\right|^{p} d V(z) \rightarrow 0$ as $\|\lambda\| \rightarrow 1-(|\alpha|=n)$;

(c) $\int_{B(\lambda, r)}\left|\frac{\partial^{n} f}{\partial z^{\alpha}}(z)\right|^{p}\left(1-\|z\|^{2}\right)^{n p-m-1} d V(z) \rightarrow 0$ as $\|\lambda\| \rightarrow 1-(|\alpha|=n)$;

(d) $\int_{\mathbf{B}_{m}}\left|\frac{\theta^{n} f}{\partial z^{\alpha}}(z)\right|^{p}\left(1-\|z\|^{2}\right)^{n p-m-1}\left(1-\left\|\varphi_{\lambda}(z)\right\|^{2}\right)^{m+1} d V(z) \rightarrow 0$ as $\|\lambda\| \rightarrow 1-(|\alpha|=n)$.

5. THE SPACE $B M O A$ AND $V M O A$

In this section we will relate some of the earlier results to the spaces $B M O A$ and 
$V M O A$, and formulate an open question. First let us recall how these spaces can be defined. For an analytic function $f$ on $\mathbf{D}$ we set

$$
\|f\|_{B M O A}=\sup _{\lambda \in \mathbf{D}}\left\|f \circ \varphi_{\lambda}-f(\lambda)\right\|_{H^{2}} .
$$

The space $B M O A$ ("Bounded Mean Oscillation," see [5]) is the set of all analytic. functions $f$ on $\mathbf{D}$ for which $\|f\|_{B M O A}<\infty$. Contained in $B M O A$ is the subspace $V M O A$ ("Vanishing Mean Oscillation"), the set of all analytic functions $f$ on $D$ for which $\left\|f \circ \varphi_{\lambda}-f(\lambda)\right\|_{H^{2}} \rightarrow 0$ as $|\lambda| \rightarrow 1-$. Paley's integral inequalities ([6], Lemma 3.2 ) and a change-of-variable give us that for every analytic function $f$ on $D$.

$$
\|f\|_{B M O A} \approx \sup _{\lambda \in \mathbf{D}}\left(\int_{\mathbf{D}}\left|f^{\prime}(z)\right|^{2}\left(1-\left|\varphi_{\lambda}(z)\right|^{2}\right) d A(z)\right)^{1 / 2},
$$

and

$$
f \in V M O A \Longleftrightarrow\left[\int_{\mathbf{D}}\left|f^{\prime}(z)\right|^{2}\left(1-\left|\varphi_{\lambda}(z)\right|^{2}\right) d A(z) \rightarrow 0 \text { as }|\lambda| \rightarrow 1-\right] .
$$

The Bloch space and $B M O A$ share many analogous properties, as do the little Bloch space and $V M O A$. The equivalence

$$
\|f\|_{\mathcal{B}} \approx \sup _{\lambda \in \mathbf{D}}\left(\int_{\mathbf{D}}\left|f^{\prime}(z)\right|^{2}\left(1-\left|\varphi_{\lambda}(z)\right|^{2}\right)^{2} d A(z)\right)^{1 / 2}
$$

in Theorem 1 should be compared with equivalence (12) for $\|f\|_{B M O A}$. Clearly, $B M O A$ is contained in the Bloch space and $V M O A$ in the little Bloch space. The above comparison leads us to the following open question:

Question. Let $0<p<\infty$ and let $f$ be an analytic function on $\mathbf{D}$. Are the following true?

(i) $f \in B M O A \Longleftrightarrow \sup _{\lambda \in \mathbf{D}} \int_{\mathbf{D}}\left|f^{\prime}(z)\right|^{p}\left(1-|z|^{2}\right)^{p-2}\left(1-\left|\varphi_{\lambda}(z)\right|^{2}\right) d A(z)<\infty$;

(ii) $f \in V M O A$

$$
\Longleftrightarrow\left[\int_{\mathbf{D}}\left|f^{\prime}(z)\right|^{p}\left(1-|z|^{2}\right)^{p-2}\left(1-\left|\varphi_{\lambda}(z)\right|^{2}\right) d A(z) \rightarrow 0 \text { as }|\lambda| \rightarrow 1-\right] \text {. }
$$

We do not know an answer to the above question. The classical results of Littlewood and Paley ([7], Theorems 5 and 6, page 54) and a change-of-variables give a partial answer, depending on $p$ : for an analytic function $f$ on $\mathbf{D}$ and $0<p \leqslant 2$ the conditions in (i) and (ii) are sufficient for containment in $B M O A$ and $V M O A$, respectively; for $2 \leqslant p<\infty$ the conditions in (i) and (ii) are necessary for $f$ to belong to $B M O A$ and $V M O A$, respectively. These implications can also be proved directly using Theorem 1. We omit the details.

The following theorem gives sufficient conditions for containment in the spaces $B M O A$ and $V M O A$. 
Theorem 9. Let $0<p<\infty, 0<\sigma<1$. Then for an analytic function $f$ on $\mathbf{D}$ we have the following two implications:

(i) $\sup _{\lambda \in \mathbf{D}} \int_{\mathbf{D}}\left|f^{\prime}(z)\right|^{p}\left(1-|z|^{2}\right)^{p-2}\left(1-\left|\varphi_{\lambda}(z)\right|^{2}\right)^{\sigma} d A(z)<\infty$ implies that $f \in$ $B M O A$;

(ii) $\int_{\mathbf{D}}\left|f^{\prime}(z)\right|^{p}\left(1-|z|^{2}\right)^{p-2}\left(1-\left|\varphi_{\lambda}(z)\right|^{2}\right)^{\sigma} d A(z) \rightarrow 0$ as $|\lambda| \rightarrow 1-$ implies that $f \in V M O A$.

Proof: Let $0<\sigma<1$. First we will prove that both statements hold for $p>2$. Let $f$ be analytic on $\mathbf{D}$. Writing $\beta=p+\sigma-2$, and using Hölder's inequality with index $p / 2$, which has conjugate index $p /(p-2)$, we have

$$
\begin{aligned}
& \int_{\mathbf{D}}\left|f^{\prime}(z)\right|^{2}\left(1-|z|^{2}\right) d A(z) \\
& \quad \leqslant\left(\int_{\mathbf{D}}\left|f^{\prime}(z)\right|^{p}\left(1-|z|^{2}\right)^{\beta} d A(z)\right)^{2 / p} \cdot\left(\int_{0}\left(1-|z|^{2}\right)^{\frac{p-z \beta}{p-2}} d A(z)\right)^{1-2 / p}
\end{aligned}
$$

thus

$$
\left(\int_{\mathrm{D}}\left|f^{\prime}(z)\right|^{2}\left(1-|z|^{2}\right) d A(z)\right)^{1 / 2} \leqslant C\left(\int_{\mathrm{D}}\left|f^{\prime}(z)\right|^{p}\left(1-|z|^{2}\right)^{\beta} d A(z)\right)^{1 / p}
$$

where $C$ is a constant depending only on $p$ and $\sigma$. Now let $\lambda \in \mathbf{D}$, and replace $f$ by its Möbius transform $f \circ \varphi_{\lambda}-f(\lambda)$. Making use of identity (5) and the changeof-variable formula (6) we see that the integral at the right hand side of the above inequality transforms into

$$
\begin{aligned}
\int_{\mathbf{D}}\left|f^{\prime}\left(\varphi_{\lambda}(z)\right)\right|^{p} & \left(\frac{1-\left|\varphi_{\lambda}(z)\right|^{2}}{1-|z|^{2}}\right)^{p-2}\left(1-|z|^{2}\right)^{\beta}\left|\varphi_{\lambda}^{\prime}(z)\right|^{2} d A(z) \\
& =\int_{\mathbf{D}}\left|f^{\prime}\left(\varphi_{\lambda}(z)\right)\right|^{p}\left(1-\left|\varphi_{\lambda}(z)\right|^{2}\right)^{p-2}\left(1-|z|^{2}\right)^{\sigma}\left|\varphi_{\lambda}^{\prime}(z)\right|^{2} d A(z) \\
& =\int_{\mathbf{D}}\left|f^{\prime}(w)\right|^{p}\left(1-|w|^{2}\right)^{p-2}\left(1-\left|\varphi_{\lambda}(w)\right|^{2}\right)^{\sigma} d A(w) .
\end{aligned}
$$

It follows that

$$
\begin{aligned}
&\left(\int_{0}\left|f^{\prime}(z)\right|^{2}\left(1-\left|\varphi_{\lambda}(z)\right|^{2}\right)\right.d A(z))^{1 / 2} \\
& \leqslant C\left(\int_{0}\left|f^{\prime}(z)\right|^{p}\left(1-|z|^{2}\right)^{p-2}\left(1-\left|\varphi_{\lambda}(z)\right|^{2}\right)^{\sigma} d A(z)\right)^{1 / p}
\end{aligned}
$$


and, in view of (12) and (13), both statements follow immediately.

If $0<p \leqslant 2$, then we have

$$
\begin{aligned}
& \int_{\mathbf{D}}\left|f^{\prime}(z)\right|^{2}\left(1-\left|\varphi_{\lambda}(z)\right|^{2}\right) d A(z) \\
& \leqslant\|f\|_{\mathcal{B}}^{2-p} \int_{\mathbf{D}}\left|f^{\prime}(z)\right|^{p}\left(1-|z|^{2}\right)^{p-2}\left(1-\left|\varphi_{\lambda}(z)\right|^{2}\right)^{\sigma} d A(z) .
\end{aligned}
$$

Since the conditions in statements (i) and (ii) imply that $f \in \mathcal{B}$ (by Theorem 1), both statements (i) and (ii) follow at once from the above inequality. This completes the proof of this theorem.

Theorem 9 and our question preceeding it should be compared with the following theorem.

ThEOREM 10. Let $0<p<\infty, 1<\eta<\infty$. Then for an analytic function $f$ on D we have:

$$
\begin{aligned}
& \text { (i) }\|f\|_{B} \approx \sup _{\lambda \in D}\left(\int_{D}\left|f^{\prime}(z)\right|^{p}\left(1-|z|^{2}\right)^{p-2}\left(1-\left|\varphi_{\lambda}(z)\right|^{2}\right)^{\eta} d A(z)\right)^{1 / p} \\
& \text { (ii) } f \in \mathcal{B}_{0} \Longleftrightarrow\left[\int_{D}\left|f^{\prime}(z)\right|^{p}\left(1-|z|^{2}\right)^{p-2}\left(1-\left|\varphi_{\lambda}(z)\right|^{2}\right)^{\eta} d A(z) \rightarrow 0\right. \\
& \quad \text { as }|\lambda| \rightarrow 1-]
\end{aligned}
$$

We omit the proof, which is obtained by minor modifications of the one given for Theorem 1. In fact, a more general formulation, involving $n$th derivatives, is possible, but since we want to stress the analogy with the previous theorem and the question preceding it we have restricted ourselves to the above formulation.

\section{REFERENCES}

[1] J.M. Anderson, J. Clunie and Ch. Pommerenke, 'On Bloch functions and normal functions', J. Reine Ang. Math. 270 (1974), 12-37.

[2] J. Arazy and S.D. Fisher, 'Some aspects of the minimal, Möbius-invariant spaces of analytic functions on the unit disc', pages 24-44, in Interpolation Spaces and Allied Topics in Analysis, edited by $M$. Cwikel and J. Peetre (Lecture Notes in Mathematics, 1070, Springer-Verlag, Berlin-New York, 1984).

[3] J. Arazy, S.D. Fisher and J. Peetre, 'Möbius invariant function spaces', J. Reine Ang. Math. 363 (1985), 110-145.

[4] S. Axler, 'The Bergman Space, The Bloch Space and Commutators of Multiplication Operators', Duke Math. J. 63 (1986), 315-332.

[5] A. Baernstein, 'Analytic Functions of Bounded Mean Oscillation', pages 3-36, in Aspects of Contemporary Complex Analysis, edited by D.A. Brannan and J.G. Clunie (Academic Press, London, 1980).

[6] J.B. Garnett, Bounded Analytic Functions (Academic Press, New York, 1981). 
[8] L.A. Rubel and R.M. Timoney, 'An extremal property of the Bloch space', Proc. Amer. Math. Soc. 75 (1979), 45-49.

[0] W. Rudin, Function Theory in the Unit Ball of $\mathrm{C}^{N}$ (Springer-Verlag, New York, 1980).

[10] R.M. Timoney, 'Bloch functions in several complex variables, I', Bull. London Math. Soc. 12 (1980), 241-267.

[11] K. Zliu, 'The Bergman Spaces, the Bloch Space, and Gleason's Problem', Trans. Amer. Math. Soc. 309 (1988), 253-268.

Department of Mathematical Sciences

University of Montana

Missoula, Montana 59801

United States of America. 